\title{
Neopterin plasma concentrations in patients with aneurysmal subarachnoid hemorrhage: correlation with infection and long-term outcome
}

\author{
Leire Azurmendi, MSc, ${ }^{1}$ Vincent Degos, MD, PhD, ${ }^{2}$ Natalia Tiberti, PhD, ${ }^{1}$ Natacha Kapandji, MD, ${ }^{2}$ \\ Paola Sanchez-Peña, MD, ${ }^{2}$ Asita Sarrafzadeh, MD, ${ }^{3}$ Louis Puybasset, MD, ${ }^{2}$ Natacha Turck, PhD, ${ }^{1}$ \\ and Jean-Charles Sanchez, PhD' ${ }^{1}$
}

\begin{abstract}
1Department of Human Protein Sciences, University of Geneva, Geneva, Switzerland; '2Department of Anaesthesiology, Pitié-Salpêtrière University Hospital, Paris, France; and 'Department of Neurosurgery, Charité-Universitätsmedizin Berlin, Germany
\end{abstract}

OBJECTIVE Aneurysmal subarachnoid hemorrhage (aSAH) is associated with high rates of mortality and morbidity. The main predictor for the poor outcome is the World Federation of Neurosurgical Societies (WFNS) scale. However, this scale does not take into account proinflammatory events, such as infection occurring after the aSAH, which could modify the long-term status of patients. The aim of this study was to evaluate neopterin as an inflammatory biomarker for outcome and infection prediction in aSAH patients.

\begin{abstract}
METHODS Plasma concentrations of neopterin were measured in 61 aSAH patients (22 male and 39 female; mean age $[ \pm$ SD] $52.8 \pm 11.8$ years) using a commercial ELISA kit. Samples were collected daily for 10 days. Outcome at 12 months was determined using the Glasgow Outcome Scale (GOS) and dichotomized as poor (GOS score 1, 2, or 3) or good (GOS score 4 or 5). Infection was determined by the presence of a positive bacterial culture.
\end{abstract}

RESULTS Patients with poor outcome at 12 months had higher concentrations of neopterin than patients with good outcome. In the same way, patients who had an infection during the hospitalization had significantly higher concentrations of neopterin than patients without infection $(p=0.001)$. Moreover, neopterin concentrations were significantly $(p<0.008)$ elevated in infected patients 2 days before infection detection and antibiotic therapy.

CONCLUSIONS Neopterin is an efficient outcome predictor after aSAH. Furthermore, it is able to differentiate between infected and uninfected patients as early as 2 days before clinical signs of infection, facilitating earlier antibiotic therapy and better management.

http://thejns.org/doi/abs/10.3171/2015.3.JNS142212

KEY WORDS aneurysmal subarachnoid hemorrhage; outcome; infection; inflammation; biomarker; neopterin; vascular disorders

$\mathrm{A}$ NEURYSMAL subarachnoid hemorrhage (aSAH) is a subtype of stroke associated with high rates of mortality and morbidity. Fifteen percent of patients die before arriving at a hospital, and $25 \%$ of deaths occur within the first 24 hours. ${ }^{34}$ Among the survivors, $30 \%$ will develop a long-term delayed neurological deficit that will affect their quality of life..$^{21,32}$

The long-term outcome partially depends on early di- agnosis and management. ${ }^{1,12}$ Therefore, if an aneurysm is detected by $\mathrm{CT}$ in a patient with aSAH, invasive treatment is indicated to prevent a second hemorrhage from the affected vessel, the most important cause of death in the first 24 hours following aSAH. ${ }^{20,25}$ Large studies, such as the International Subarachnoid Aneurysm Trial (ISAT), have investigated whether neurosurgical clipping or endovascular coiling improve the long-term outcome. It has been

ABBREVIATIONS aSAH = aneurysmal subarachnoid hemorrhage; $\mathrm{AUC}=$ area under the ROC curve; $\mathrm{DCl}=$ delayed cerebral ischemia; $\mathrm{EVD}=$ external ventricular drain; GCS = Glasgow Coma Scale; GOS = Glasgow Outcome Scale; NSE = neuron-specific enolase; ROC = receiver operating characteristic; ROS = reactive oxygen species; S100 $\beta=$ S100 calcium binding protein $\beta ;$ WBC $=$ white blood cell; WFNS = World Federation of Neurosurgical Societies .

SUBMITTED September 24, 2014. ACCEPTED March 10, 2015.

INCLUDE WHEN CITING Published online September 25, 2015; DOI: 10.3171/2015.3.JNS142212. 
shown, however, that the outcome depends mainly on unmodifiable factors present at admission and on secondary complications rather than the choice of treatment., 30,25

Until recently, the most accepted criteria for predicting the outcome of aSAH have been increased age and results of clinical and radiological assessment scales for evaluation of the patient's neurological state at hospital admission, such as the Fisher grade, which measures the severity of the hemorrhage, ${ }^{17}$ and the World Federation of Neurosurgical Societies (WFNS) scale, which uses the Glasgow Coma Scale (GCS) and assessment of focal neurological deficits to establish the severity of symptoms. . $4,17,28^{-1}$

Interest in the measurement of CSF and blood biomarkers has increased in the last few years because the ability to monitor patients throughout hospitalization may lead to important improvements in their management. Increased concentrations of S100 calcium binding protein$\beta$ (S100 $\beta$ ), C-reactive protein, adhesion and matrix molecules, and vasogenic and cardiac markers have been found in aSAH patients with poor outcomes, but the limited accuracy of most of these markers has hampered their use in clinical practice. . $^{13,27,36,38}$

Neopterin, a catabolic product of GTP (guanosine-5-triphosphate) produced by human monocytes-macrophages upon stimulation with interferon- $\gamma$, has been reported as an outcome and infection biomarker for several inflammatory diseases. ${ }^{6,19,35}$ Moreover, Mathiesen et al. showed that the CSF and plasma concentrations of neopterin were higher in patients suffering from aSAH than in controls. The use of neopterin to predict patient outcomes was also studied, but no association was found. ${ }^{23}$

We hypothesized that inflammation and infection after the hemorrhagic event have important effects on outcome, ${ }^{11}$ and we evaluated neopterin as a biomarker of inflammation and infection that may be useful in predicting outcomes in aSAH patients.

\section{Methods}

\section{Patients and Sample Collection}

The aSAH patients included in the present study were hospitalized at the Pitié-Salpêtrière Hospital of Paris (France) between July 2004 and April 2008. Aneurysmal SAH was confirmed angiographically and by CT. The study was approved by the local ethics committee (Comité de Protection des Personnes, Pitié-Salpêtrière, Paris, France). All patients or their surrogates signed a written informed consent.

Patient neurological status was evaluated on admission to the intensive care unit by use of the WFNS scale. Depending on the location and the size of the aneurysm, the aneurysm was not treated, was treated by surgical clipping, or was treated by endovascular coil embolization. More details on the clinical monitoring and treatment have been previously described by Turck et al. ${ }^{36}$

Among 198 consecutively hospitalized patients, 137 were excluded from the present study due to 1) presence of more than 1 hemorrhagic event, 2) admission to the hospital more than 48 hours after the onset of symptoms, 3 ) missing clinical information, or 4) insufficient sample volume to perform our assays. Sixty-one patients were then finally included in this study (Fig. 1).

Plasma samples were collected the day of arrival at the hospital and subsequently every day for 10 consecutive days (Day 1-Day 10).

The Glasgow Outcome Scale (GOS) was used to assess patient outcomes by telephone interview 12 months after the hemorrhagic event. Depending on the grade of dependence, the outcome was classified as poor (GOS score 1,2, or 3 ) or good (GOS score 4 or 5) by a doctor who had not participated in the initial care.

During hospitalization, patient infection status was defined daily based on the criteria established by the Interna-

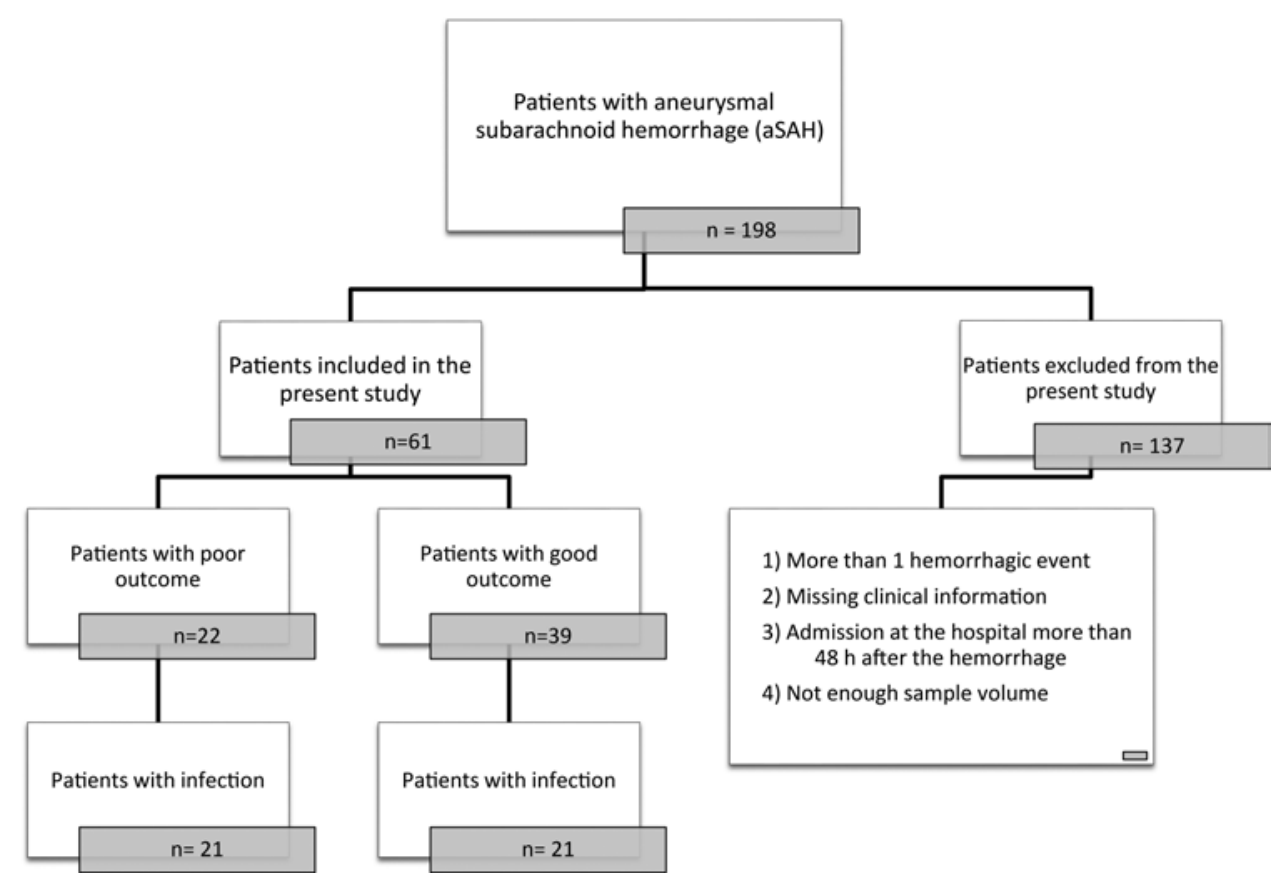

FIG. 1. Inclusion criteria flowchart for the population investigated in this study. 
tional Sepsis Forum Consensus Conference on Definitions of Infection in the Intensive Care Unit. ${ }^{4}$ When there was a suspicion of infection based on systematic clinical and biological criteria, bacteriological samples were obtained. Antibiotic therapy was started as soon as there was suspicion of infection and was readjusted once the results of the cultures were obtained. The first day of antibiotic therapy in infected patients was designated as $\mathrm{T}-0$, the day before treatment as $\mathrm{T}-1$, and 2 days before treatment as $\mathrm{T}-2$.

\section{Neopterin and S100 $\beta$ Assays}

Neopterin concentrations in plasma samples were measured using a commercially available enzyme-linked immunosorbent assay (ELISA) kit according to the manufacturer's instructions (BRAHMS GmbH).

S100 $\beta$ concentrations were determined with an immunoluminometric sandwich assay on an LIA-mat 300 analyzer (Byk-Sangtek France Laboratories) using manufacturer's reagents. ${ }^{2}$ Technical variability was assessed using internal quality controls (coefficient of variation $\leq 20 \%$ ).

Concentrations of both molecules were measured once per day during the first 10 days of hospitalization in order to evaluate their capacity to act as outcome and infection markers.

\section{Statistical Analysis}

Statistical analyses were performed with SPSS software (version 21, SPSS Inc.). The Shapiro-Wilk test was used to test deviations from Gaussian distribution of age, neopterin concentration, and S100 $\beta$ concentration. Age was normally distributed, and a parametric test was performed to compare the differences between the ages of patients with good versus poor outcome. Because neopterin and $\mathrm{S} 100 \beta$ concentrations did not follow a normal distribution, the Mann-Whitney U-test was used for statistical comparisons between 2 unpaired groups and the Friedman test for comparisons of paired groups. Fisher's exact test and the chi-square test were used to assess whether the groups of patients with good or poor outcome differed significantly with respect to sex, WFNS grade, modified Fisher scale grade, presence of convulsions, use of external ventricular drain (EVD), presence of hydrocephalus, and treatment. Univariate and multivariate analyses were performed to assess the association between variables. In a first analysis, the 12-month GOS score was set as the dependent variable and the WFNS grade, S100 $\beta$ concentration, neopterin concentration, and presence of infection as confounders. In a second analysis, the presence/absence of infection was set as the dependent variable and sex, WFNS grade, blood white blood cell (WBC) count, neopterin concentration, and EVD use were set as confounders. The model was validated using the bootstrap method. Categorical data were dichotomized according to the criteria of the demographic characteristics table. Longitudinal data were also dichotomized according to the best cutoff obtained from area under the receiver operating characteristic (ROC) curve (AUC) analysis.

All statistical tests were bilateral, and a p value $<0.05$ was considered statistically significant. When multiple comparisons were done, Bonferroni correction of the $\mathrm{p}$ values was applied. Sample size estimation was calculated to obtain a power of $90 \%$ and a 2 -sided error of $5 \% .{ }^{30}$

Receiver operating characteristic curves were calculated for WFNS grade at Day 1 and for neopterin and S100 $\beta$ concentrations from Day 1 to Day 10 . AUC values, specificity, sensitivity, and 95\% CIs were calculated with the pROC package for S+, version 8.1. (TIBCO Software Inc.). ${ }^{30} \mathrm{~A}$ cutoff value corresponding to the best combination of specificity and sensitivity was obtained at each time point for both markers. The Youden index $(\mathrm{J})$ was obtained using the following formula: $\mathrm{J}=(\mathrm{SE} \%+\mathrm{SP} \%)-$ 100 , where SE is sensitivity and SP is specificity.

\section{Results \\ Demographic Characteristics}

The demographic characteristics of the 61 patients included in this study are summarized in Table 1. Most of the patients were women (64\%), and the patients' mean age $( \pm \mathrm{SD})$ was $52.8 \pm 11.8$ years. In this cohort, $61 \%$ of the patients had no motor deficit at admission to the hospital (WFNS grade 1 or 2), and 75\% were treated with embolization. Angiographic vasospasm developed in $38 \%$ of the patients and infection in $69 \%$.

\section{Prediction of Outcome According to Neopterin and S100 $\beta$ Plasma Concentrations}

At 12 months after hospital admission there were no significant differences between patients with good and those with poor outcomes with respect to the modified Fisher scale grade or the development of delayed cerebral ischemia (DCI), seizures (data not shown), or angiographic vasospasm. Nevertheless, univariate analyses showed that even though our study group of patients with aSAH included more women than men, the number of men who had a poor outcome $(n=12,54.5 \%)$ was greater than the number of women $(n=10,45.5 \%)$. WFNS grade and GCS score were highly associated with poor outcome at 12 months ( $p<0.0001)$. Furthermore, the intervention technique (clipping or coiling) appeared to play an important role in the long-term outcome, as all the patients whose aneurysms were not treated had a poor outcome at 12 months $(p=0.005)$. Finally, most of the patients with a poor outcome after hospitalization (21 of 22) developed an infection during hospitalization, which strengthened the association between outcome and the presence of infection $(\mathrm{p}=0.007$, OR 18).

To evaluate the utility of neopterin and $\mathrm{S} 100 \beta$ for predicting 12-month outcome after aSAH, the concentrations were measured every day. Patients were classified according to poor (GOS score 1, 2, or 3) or good (GOS score 4 or 5) outcome at 12 months. Results are shown in Fig. 2. Patients with poor outcomes showed significantly higher plasma concentrations of neopterin and $\mathrm{S} 100 \beta$ than patients with good outcome. The $\mathrm{S} 100 \beta$ concentrations were elevated from the day of hospital admission and remained elevated during the whole period of measurement, whereas significantly higher concentrations of neopterin were found from 3 days after hospitalization (Day 3) onward. Moreover, in the case of neopterin, a progressive increase in concentrations was observed. 
TABLE 1. Comparison of demographic and clinical characteristics of 61 aSAH patients stratified by outcome at 12 months*

\begin{tabular}{|c|c|c|c|c|}
\hline \multirow[b]{2}{*}{ Characteristic } & \multirow[b]{2}{*}{ Total $(n=61)$} & \multicolumn{3}{|c|}{ Outcome at 12 Mos } \\
\hline & & Good $(n=39)$ & Poor $(n=22)$ & p Value $†$ \\
\hline Sex & & & & 0.03 \\
\hline Male & $22(36.1 \%)$ & $10(25.6 \%)$ & $12(54.5 \%)$ & \\
\hline Female & $39(63.9 \%)$ & $29(74.4 \%)$ & $10(45.5 \%)$ & \\
\hline Age in yrs & & & & $0.875 \ddagger$ \\
\hline Mean (SD) & $52.8(11.8)$ & $52.3(12.5)$ & $53.8(10.5)$ & \\
\hline WFNS grade & & & & $<0.0001$ \\
\hline 1 or 2 & $37(60.7 \%)$ & $32(82.1 \%)$ & $5(22.7 \%)$ & \\
\hline 3,4, or 5 & $24(39.3 \%)$ & $7(17.9 \%)$ & $17(77.3 \%)$ & \\
\hline GCS score & & & & $<0.0001$ \\
\hline$<9$ & $15(24.6 \%)$ & $2(5.1 \%)$ & $13(59.1 \%)$ & \\
\hline $9-12$ & $7(11.5 \%)$ & $3(7.7 \%)$ & $4(18.2 \%)$ & \\
\hline$\geq 13$ & $39(63.9 \%)$ & $34(87.2 \%)$ & $5(22.7 \%)$ & \\
\hline Vasospasm & & & & 0.871 \\
\hline Yes & $23(37.7 \%)$ & $15(38.5 \%)$ & $8(36.4 \%)$ & \\
\hline No & $38(62.3 \%)$ & $24(61.51 \%)$ & $14(63.6 \%)$ & \\
\hline Treatment & & & & 0.005 \\
\hline Surgery & $11(18 \%)$ & $5(12.8 \%)$ & $6(27.3 \%)$ & \\
\hline Embolization & $46(75.4 \%)$ & $34(87.2 \%)$ & $12(54.5 \%)$ & \\
\hline No treatment & $4(6.6 \%)$ & $0(0 \%)$ & $4(18.2 \%)$ & \\
\hline EVD & & & & $<0.0001$ \\
\hline Yes & $37(60.6 \%)$ & $16(41 \%)$ & $21(95.5 \%)$ & \\
\hline No & $24(39.4 \%)$ & $23(59 \%)$ & $1(4.5 \%)$ & \\
\hline Infection & & & & $<0.0001$ \\
\hline Yes & $42(68.9 \%)$ & $21(53.8 \%)$ & $21(95.5 \%)$ & \\
\hline No & $19(31.1 \%)$ & $18(46.2 \%)$ & $1(4.5 \%)$ & \\
\hline
\end{tabular}

To evaluate the performance of these 2 variables for the prediction of patient outcomes, ROC curves were used. Detailed results are shown in Table 2 and Figs. 3 and 4. For neopterin, the best discrimination was found 5 days after hospitalization: AUC 84.2\% (95\% CI 71.9\%-94.1\%) for a cutoff concentration of $12.7 \mathrm{nmol} / \mathrm{L}$, with specificity and sensitivity values of $74.4 \%$ and $86.4 \%$, respectively $(\mathrm{J}=60.8)$. For $\mathrm{S} 100 \beta$, the best discrimination was found 10 days after hospitalization: AUC 86.9\% (95\% CI $73.5 \%-97.3 \%$ ) for a cutoff concentration of $0.2 \mu \mathrm{g} / \mathrm{L}$, with specificity and sensitivity values of $86.4 \%$ and $88.2 \%$, respectively.

Taking into account the best cutoff values obtained from the ROC analysis (AUC), we dichotomized the longitudinal variables in univariate and multivariate regression analyses. Univariate analysis showed that sex, presence of infection, and WFNS grade, as well as the concentrations of neopterin and S100 $\beta$, were associated with outcome at 12 months. In multivariate analysis, however, only neopterin concentration and WFNS grade displayed a relationship with the patients' long-term status (Table 3).

The combination of WFNS grade at admission to the
hospital-AUC $89 \%$ (95\% CI 81.3\%-96.7\%), specificity $87.2 \%$ and sensitivity $77.3 \%(\mathrm{~J}=64.5)$ - and neopterin concentration at Day 5 dramatically improved the overall accuracy of outcome prediction, reaching a Youden index (J) of 75.5 .

\section{Infection Prediction According to Plasma Neopterin Concentration and WBC Count}

Infection in aSAH patients can produce systemic inflammatory response syndrome, with associated elevated body temperature, elevated heart rate, tachypnea, and leukocytoses. Because these symptoms appeared to be important factors affecting prognosis after aSAH $(\mathrm{p}=0.001)$, we evaluated whether WBC counts and neopterin concentrations were significantly different in patients with $(\mathrm{n}=$ $42)$ and without infection $(n=19)$.

We first evaluated the capacity of these variables to help us decide whether or not antibiotic therapy should be initiated. We found that the concentrations of neopterin obtained on the first day of antibiotic treatment for patients who developed an infection were significantly greater than concentrations obtained at the corresponding time point 

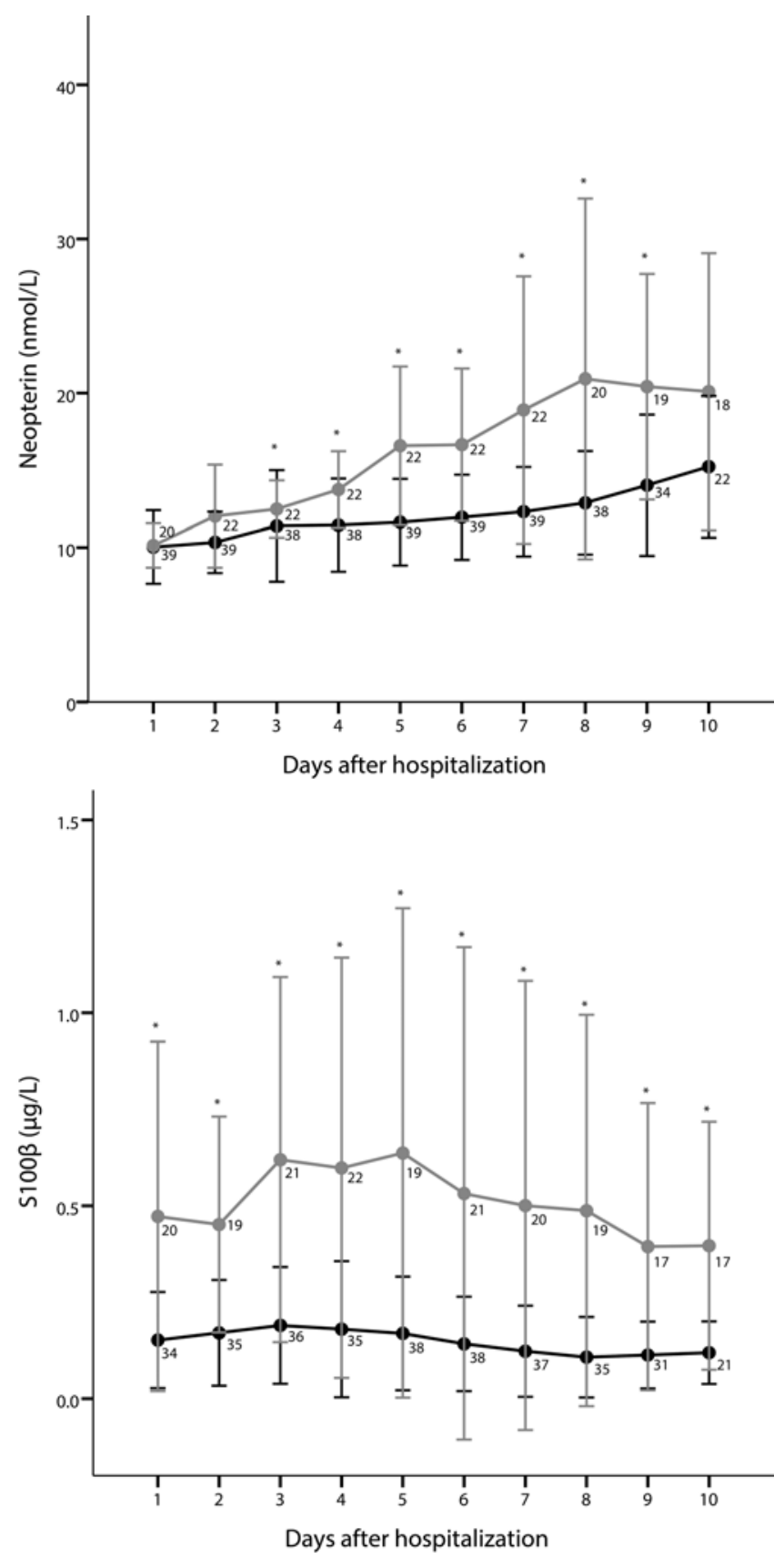

FIG. 2. Kinetics of neopterin (upper) and $S 100 \beta$ (lower) concentrations from the day of hospital admission to 10 days thereafter with patients grouped according to outcome. The mean neopterin and $\mathrm{S} 100 \beta$ concentrations are indicated by solid black circles for the patients with poor 12-month outcome (GOS score 1-3) and by solid gray circles for those with good outcome (GOS score 4 or 5). Error bars in corresponding colors represent the standard deviations. The numbers on the graphs represent the number of patients tested at each time point. Comparison between 2 groups was performed using the Mann-Whitney U-test. * $p<$ 0.05, after Bonferroni correction.

in those who did not develop an infection (see Discussion) (Fig. 5). The Youden index performance, used to differentiate between these 2 groups, reached a value of $\mathrm{J}=39.59$. In contrast, the WBC count did not differ significantly between the 2 groups (see Table 5 and Fig. 6).
Because neopterin concentration appeared to be an efficient biomarker for infection, we evaluated its performance throughout the entire hospitalization. Neopterin concentrations were significantly higher in infected patients from 3 days after hospitalization onward up to 10 days (Fig. 7). The performance of this biomarker in establishing this differentiation was most effective at Day 3 (AUC 76.7\%), which corresponded in most of the cases with 2 days before the start of infection treatment (Table 5 and Fig. 8).

To evaluate whether this increase in neopterin concentrations in infected patients was correlated with the appearance of infection, univariate analyses were performed using values obtained at Day 5, which represented the mean day of infection detection (i.e., the mean of the numbers of days of hospitalization at which infection was detected). Univariate analysis demonstrated that there was a relationship between the presence of an infection and high concentrations of neopterin, poor neurological state at admission to the hospital, and the placement of an EVD. There was, however, no relation between the WBC count and the development of infection. When we tested the same parameters in multivariate analyses, neopterin remained an independent factor for the presence of infection in aSAH patients (Table 4).

\section{Discussion}

Long-term outcome after aSAH is a major clinical issue, and the rates of mortality and morbidity are high. ${ }^{31}$ The prediction of 12-month outcome as well as the detection of complications during the hospitalization may help physicians to provide better care for aSAH patients and better inform family members of what to expect during the months to come..$^{15}$

Several unmodifiable factors, such as increased age or the level of consciousness of the patient at hospital admission, have been proposed and used as the best univariate predictors of outcome..$^{17}$ Among these, we found that the level of consciousness (GCS score) and focal neurological deficit (WFNS score) were the strongest predictors ( $p<$ 0.001 ). However, the failure of these neurological assessment scales to address complications occurring during hospitalization has highlighted the need for blood biomarker identification.

The calcium-binding protein $\mathrm{S} 100 \beta$ has been one of the most commonly used prognostic biomarkers in various conditions associated with brain damage, such as traumatic brain injury, stroke, and aSAH.$^{27}$ However, the use of this biomarker in clinical practice has been impeded by limitations in its sensitivity and specificity. Mathiesen et al. evaluated the capacity of neopterin to act as a biomarker for outcome prediction in aSAH patients, but no association was found between neopterin concentrations and patient outcomes. ${ }^{23}$ In the present study, however, we found that increased concentrations of neopterin predicted poor outcome at 12 months in 61 patients followed for 10 days after aSAH. Although the concentrations of neopterin followed the same kinetics in both studies, the low number of patients included in the prior analysis may have been the cause of this difference in outcome determina- 


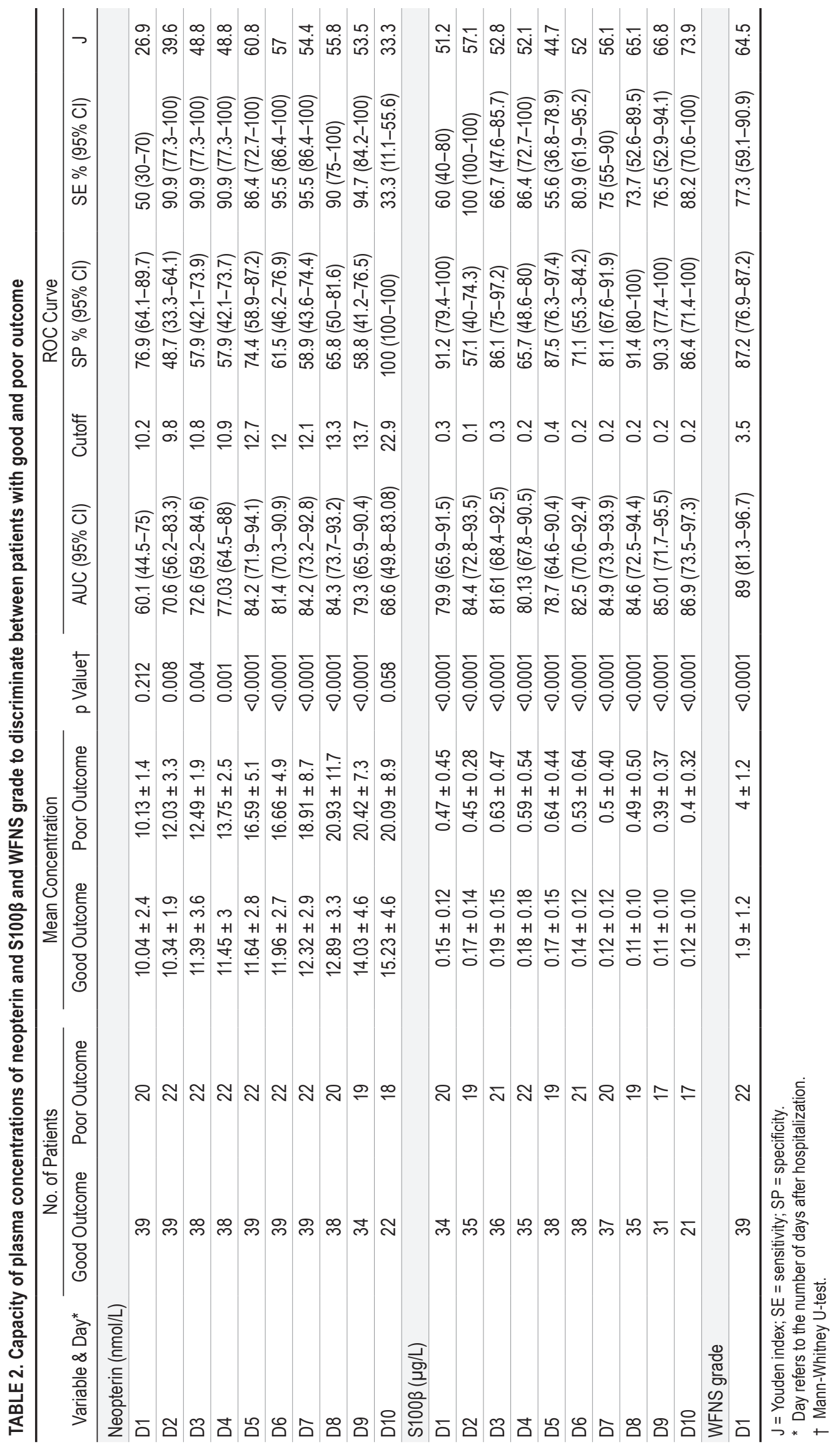



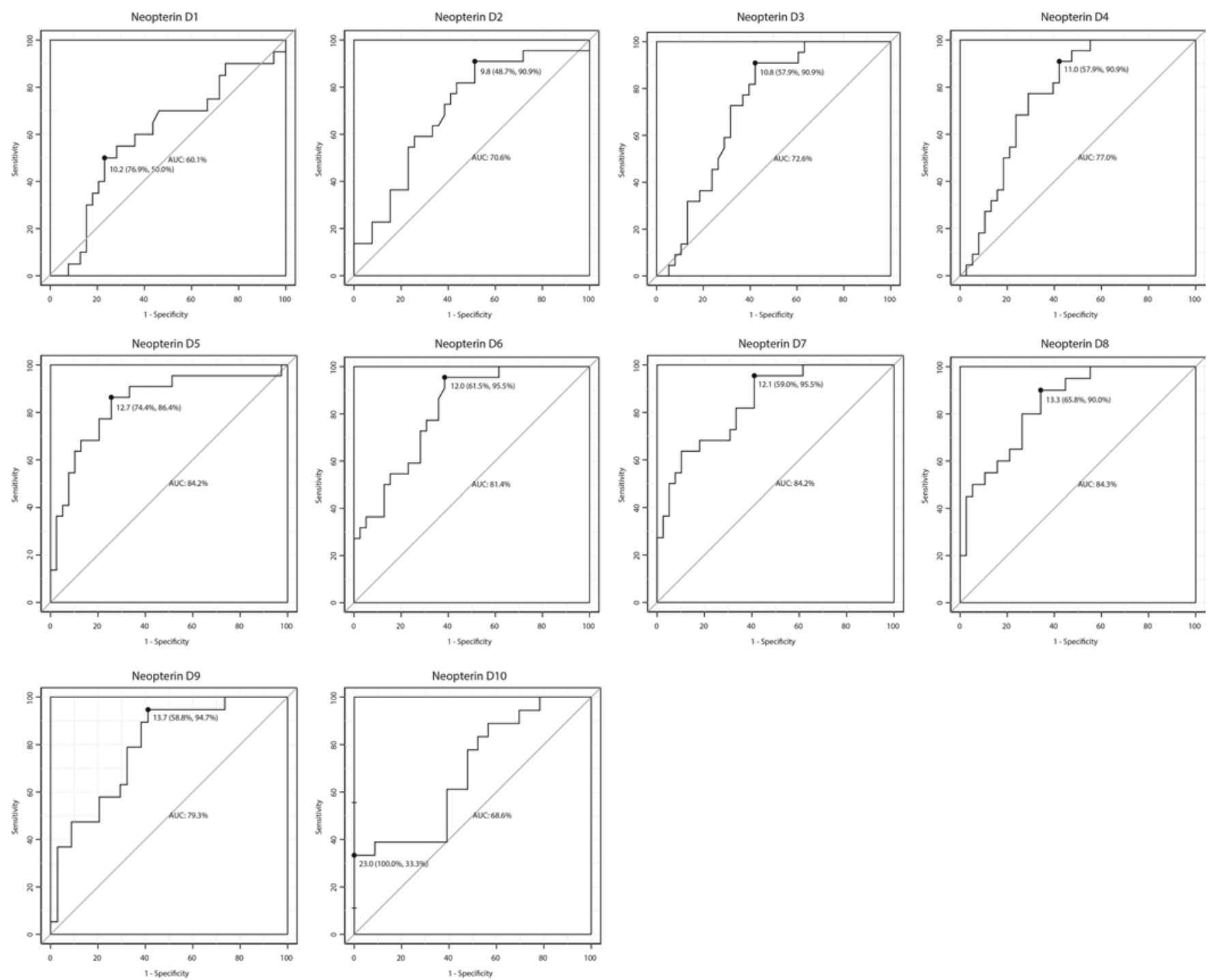

FIG. 3. ROC curves for neopterin representing the ability to differentiate between patients with poor and those with good outcomes from the day of hospital admission (Day 1 [D1]) through the 10th day of hospitalization.

tion. The performance of neopterin in differentiating between patients with good and poor outcomes is quite similar to that of S100 $\beta$ or WFNS. Nevertheless, the combination of neopterin with WFNS data substantially increases the total values for sensitivity and specificity, reaching a Youden index value $(\mathrm{J})$ of 75.5 . This fact highlights the finding that although the impact of initial hemorrhage is a key factor affecting outcome, monitoring the patient during the acute phase is also important for improving the patient's outcome.

There are 2 major advantages of neopterin over already established prognostic methods. First, neopterin is an earlier biomarker than S100 $\beta$, making it possible to identify, only 5 days after hospitalization, patients who are at higher risk of inflammatory complications. This allows the application of treatment before complications appear, increasing the level of care and consequently decreasing the long-term deterioration risk.

The second advantage is that neopterin can function as a biomarker for complications such as infection or DCI that are not addressed by the currently used clinical scales. These events must be considered during the process of biomarker discovery in aSAH, because they appear to be the main causes of pathophysiological worsening.

After the hemorrhagic event, the blood clot in the subarachnoid space leads to recruitment of adhesion molecules at the surface of endothelial cells..$^{5,22,24,29}$ Immunological cells such as neutrophils and macrophages phagocytize the red blood cells and degranulate between 2 and 4 days after activation, releasing a large quantity of intracellular endothelins and reactive oxygen species (ROS). ${ }^{26}$ The release of ROS causes a decrease in vasodilatation, leading to cerebral vasoconstriction and the development of angiographic vasospasm and DCI ${ }^{37}$ Because vasospasm and DCI are associated with important adverse effects on quality of life, many studies have been conducted to investigate their underlying mechanisms. However, other inflammatory events that play important roles in the long- 

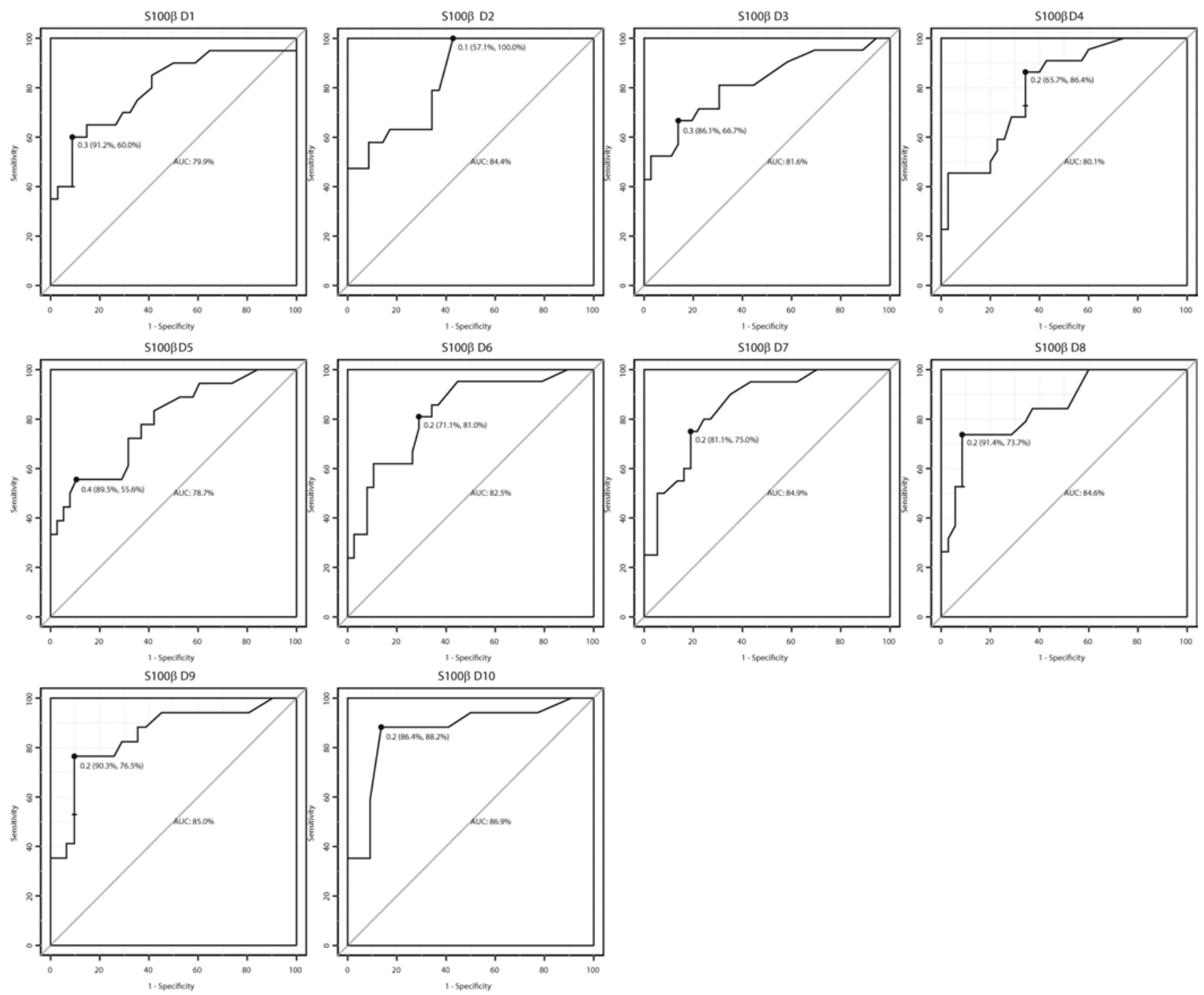

FIG. 4. ROC curves for $S 100 \beta$ representing the ability to differentiate between poor and good outcome patients from the day of hospital admission (Day 1 [D1]) through the 10th day of hospitalization.

TABLE 3. Univariate and multivariate analysis of different factors for predicting outcome at 12 months after aSAH*

\begin{tabular}{lcc}
\hline \multicolumn{1}{c}{ Analysis \& Factor } & $p$ Value & OR $(95 \% \mathrm{Cl})$ \\
\hline Univariate analysis & & \\
\hline Sex & $\mathbf{0 . 0 2 7}$ & $0.287(0.09-0.87)$ \\
\hline WFNS grade & $<\mathbf{0 . 0 0 0 1}$ & $15.54(4.28-56.44)$ \\
\hline S100ß $(<0.4 \mu \mathrm{g} / \mathrm{L})$ & $\mathbf{0 . 0 2 6}$ & $3.71(1.17-11.8)$ \\
\hline Neopterin $(12.7 \mathrm{nmol} / \mathrm{L})$ & $<\mathbf{0 . 0 0 0 1}$ & $18.37(4.46-75.52)$ \\
\hline Infection & $\mathbf{0 . 0 0 7}$ & $17.8(2.2-142.7)$ \\
\hline Multivariate analysis & & \\
\hline WFNS grade & $\mathbf{0 . 0 0 1}$ & $12.91(2.79-59.68)$ \\
\hline$\quad$ Neopterin (12.7 nmol/L) & $\mathbf{0 . 0 0 1}$ & $15.34(3.03-77.77)$ \\
\hline * Data obtained at Day 5 in a total of 61 cases were analyzed. The dichoto- \\
mization of longitudinal data was made according to the cutoff obtained in the \\
AUC analysis at Day 5; the applied values are written next to the variable. Bold \\
type indicates statistical significance.
\end{tabular}

TABLE 4. Univariate and multivariate analysis of different factors for predicting the presence of infection*

\begin{tabular}{llc}
\hline \multicolumn{1}{c}{ Analysis \& Factor } & $p$ Value & OR $(95 \% \mathrm{Cl})$ \\
\hline Univariate analysis & & \\
\hline Sex & 0.29 & $0.53(0.16-1.73)$ \\
\hline WFNS grade & $\mathbf{0 . 0 0 4}$ & $21.8(2.66-178.53)$ \\
\hline WBC $\left(10.9 \times 10^{6} \mathrm{cells} / \mathrm{mm}\right)$ & 0.18 & $2.75(0.63-12.1)$ \\
\hline Neopterin $(12.7 \mathrm{nmol} / \mathrm{L})$ & $\mathbf{0 . 0 0 2}$ & $8.66(2.17-34.49)$ \\
\hline EVD & $<\mathbf{0 . 0 0 0 1}$ & $13.75(3.65-51.81)$ \\
\hline Multivariate analysis & & \\
\hline WFNS grade & 0.099 & $6.88(0.69-68.29)$ \\
\hline Neopterin (12.7 nmol/L) & $\mathbf{0 . 0 3}$ & $5.63(1.13-28.06)$ \\
\hline EVD & $\mathbf{0 . 0 1}$ & $6.97(1.55-31.37)$ \\
\hline * Data obtained at Day 5 in a total of 61 cases were analyzed. The dichoto- \\
mization of longitudinal data was made according to the cutoff obtained in the \\
AUC analysis at Day 5 ; the applied values are written next to the variable. Bold \\
type indicates statistical significance.
\end{tabular}




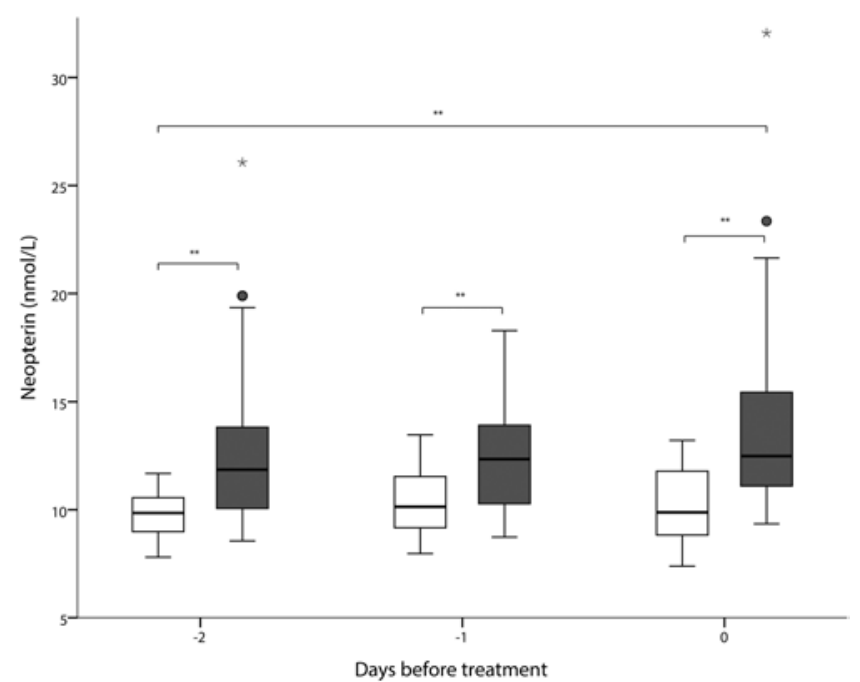

FIG. 5. Box-and-whiskers plots showing the concentrations of neopterin 2 days before initiation of antibiotic therapy, 1 day before initiation of antibiotic therapy, and the day that antibiotic therapy was initiated, with patients stratified by the presence (gray boxes, $n=35$ ) or absence (white boxes, $n=16$ ) of infection. The boxes represent interquartile ranges, the dark horizontal lines represent medians, the whiskers represent the adjacent values $(\mathrm{Q} 1-[1.5 \times \mathrm{IQR}]$ to $\mathrm{Q} 3+[1.5 \times \mathrm{IQR}]$, where IQR is interquartile range), and the outliers are shown by solid circles. Comparisons between the 2 groups were performed using the Mann-Whitney U-test. The Friedman test and Dunn posttest were used to compare the mean rank concentrations of neopterin 2 days before treatment and the day of treatment. Significance is reported after Bonferroni correction. * $p$ $<0.05,{ }^{* \star} p<0.01,{ }^{* \star *} p<0.001$.

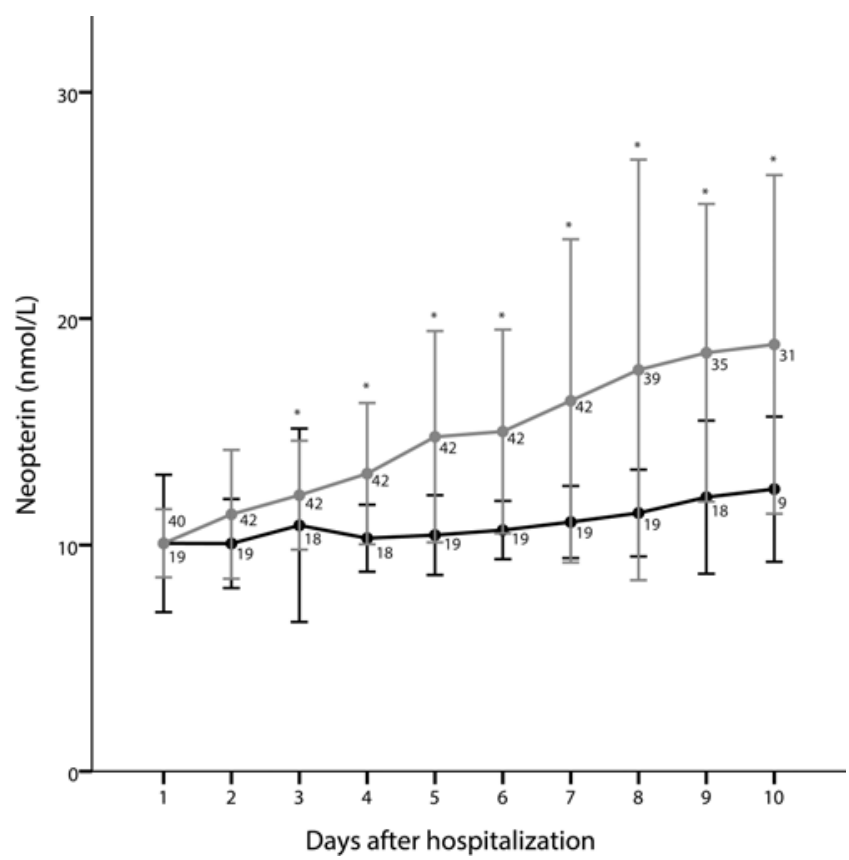

FIG. 7. Kinetics of neopterin concentration from the day of hospital admission to Day 10, with patients grouped according to the presence or absence of infection. The mean neopterin concentration is shown by solid black circles for the patients who had infection and by solid gray circles for those without infection. Error bars in corresponding colors represent the standard deviations. The numbers on the graphs represent the number of patients tested at each time point. Comparison between 2 groups was performed using the Mann-Whitney U-test. ${ }^{*} p<$ 0.05, after Bonferroni correction.
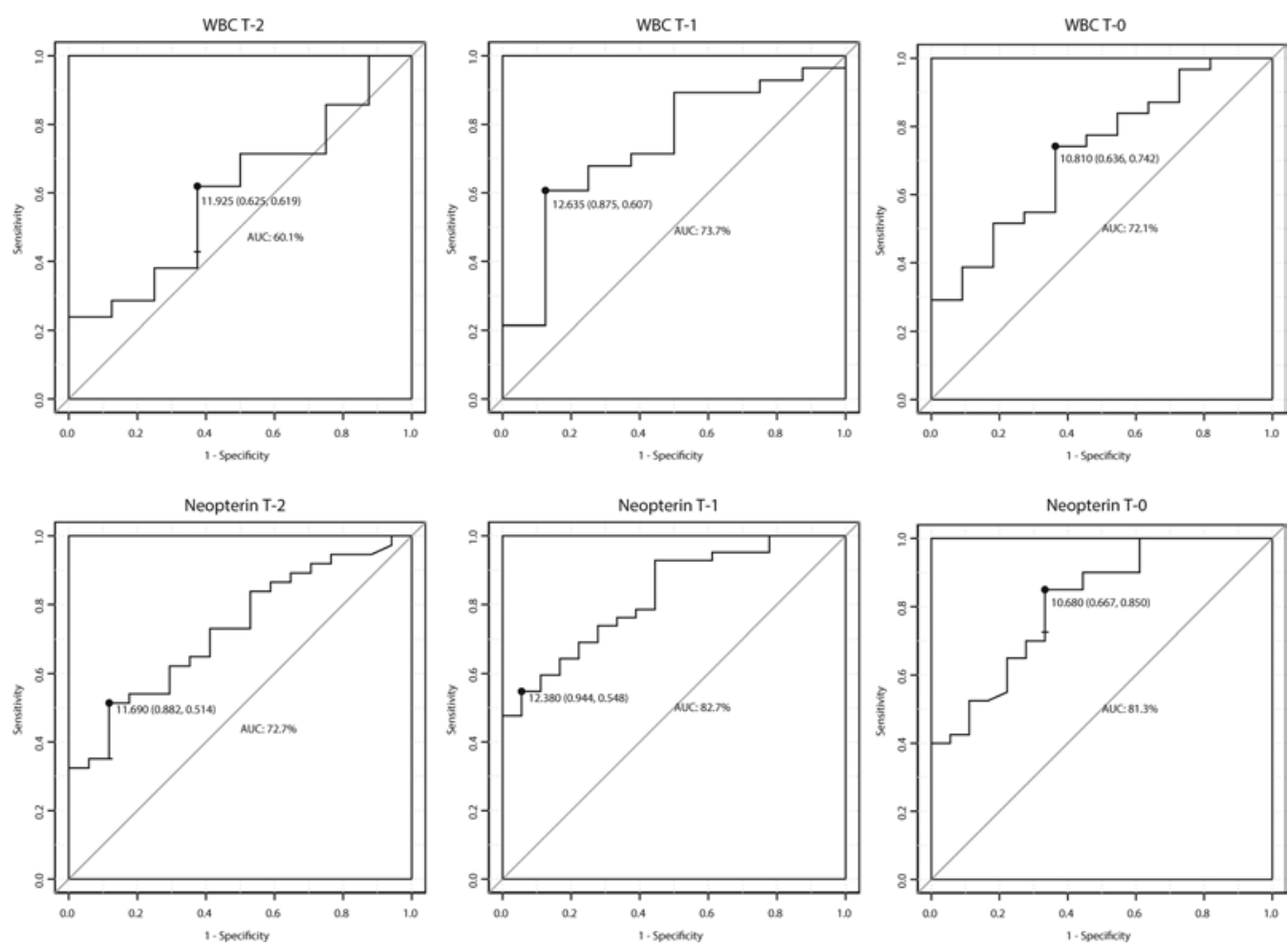

FIG. 6. ROC curves for neopterin and WBC representing the ability to differentiate between infected and uninfected patients the day antibiotic therapy was initiated ( $\mathrm{T}-0), 1$ day before $(\mathrm{T}-1)$, and 2 days before $(\mathrm{T}-2)$. 


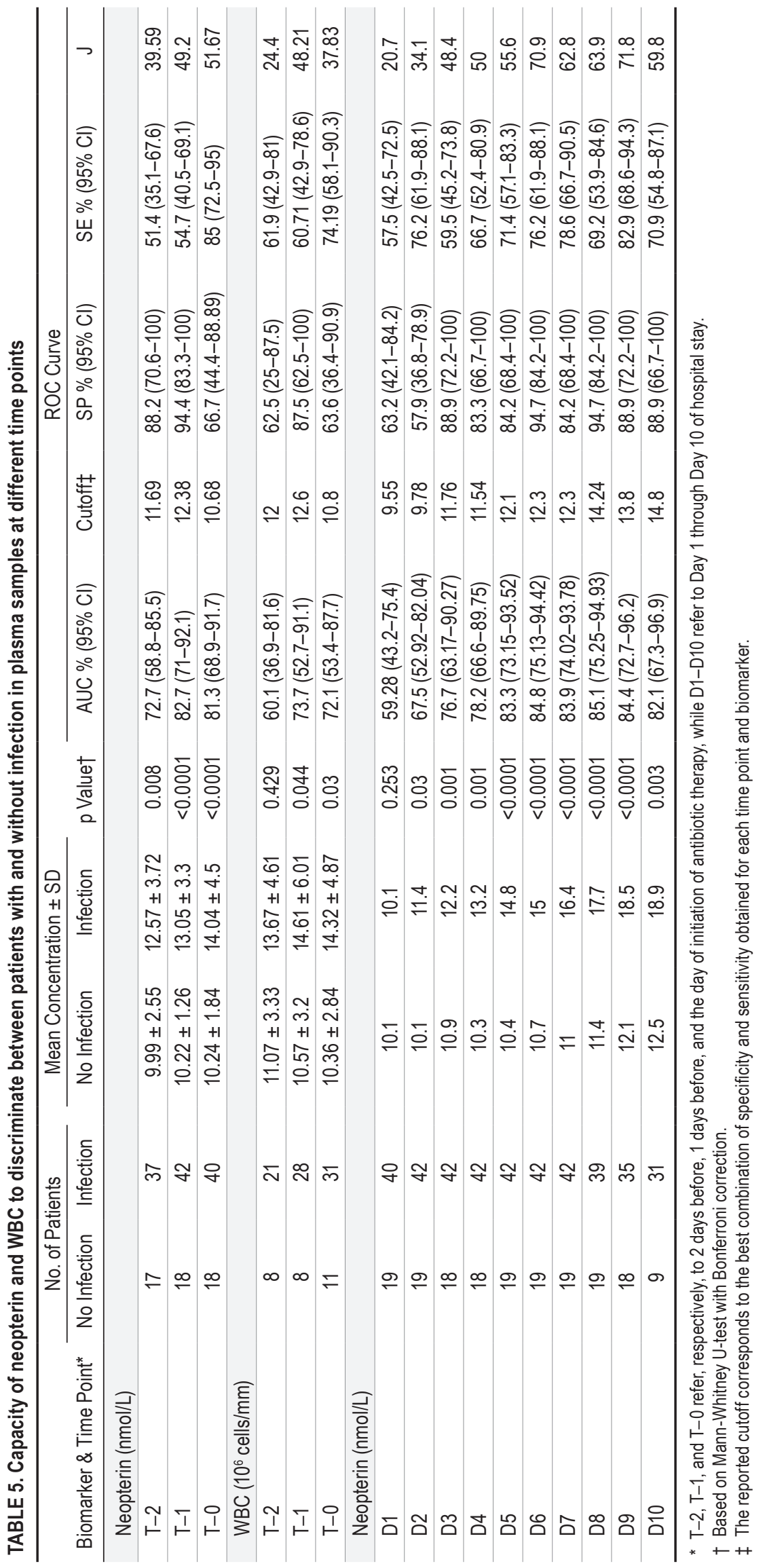



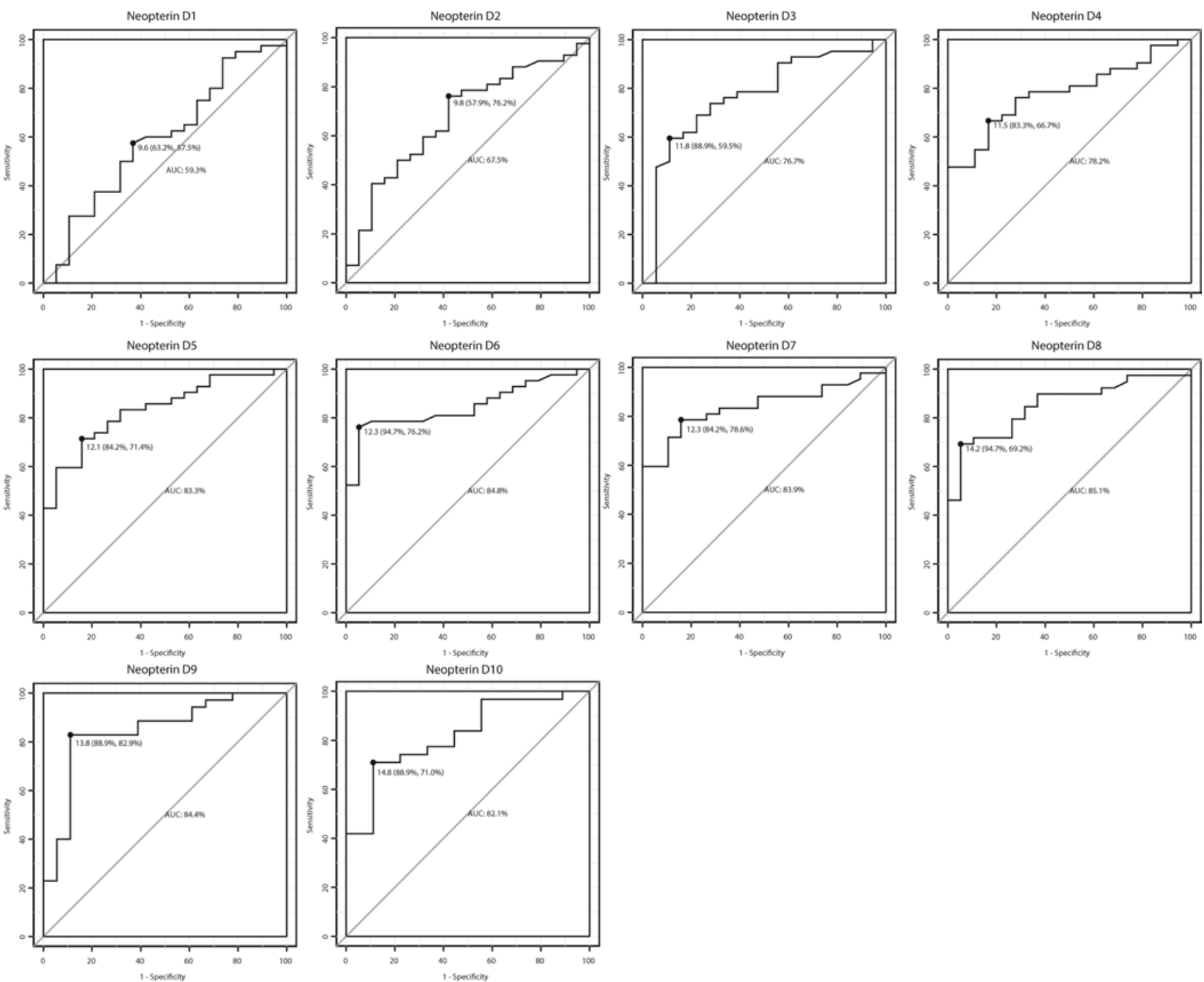

FIG. 8. ROC curves of neopterin representing the ability to differentiate between infected and uninfected patients from the admission to the hospital to 10 days after.

term condition of aSAH patients, such as infections, have not been adequately investigated..$^{8,10}$

In our study, we focused our attention on infection because infections developed during hospitalization in $95 \%$ of the patients with poor outcomes, ${ }^{10}$ indicating that early detection and treatment of bacterial infections may improve patient outcomes. In addition, the discrimination of infection from other pathologies with similar presentations should decrease the amount of unnecessary antibiotic use, avoiding associated resistance, toxicity, and allergic reactions. ${ }^{16,33}$

The WBC count is used as a reference value to detect several inflammatory diseases. However, in aSAH patients the WBC count cannot be used for this purpose because of the leukocytosis produced by the blood clot. To our knowledge, this is the first reported study showing that a marker of inflammation able to predict long-term outcome after aSAH is also correlated with the appearance of infection. Neopterin concentrations, which indicated overexpression of this biomarker in all 61 patients included in this study, enabled us to significantly differentiate between patients who developed an infection after aSAH and patients who did not. When comparing the concentrations before the detection of the infection, we found that concentrations of neopterin were already significantly different 2 days before the positive bacterial culture.

These results suggest that neopterin may be useful in clinical practice as a screening test to trigger earlier bacteriological studies. Based on our study results, the ideal day to measure neopterin concentrations seems to be 3 days after admission to the hospital; this is approximately 2 days before the detection of infection by bacterial culture. At this time point, we found that a cutoff value of $11.8 \mathrm{nmol} / \mathrm{L}$ would allow correct classification in $77 \%$ of the cases with respect to whether infection will develop or not. However, several critical issues should be further evaluated regarding the use of this biomarker. The association between infection and outcome must be clarified to confirm that prevention of infection leads to improvement in patient outcomes. In addition, the exact time or sequen- 
tial times to measure the neopterin concentrations, as well as the best cutoff values to trigger initiation of treatment, must be defined.

Thus far, we have not been able to confirm that neopterin is a direct diagnostic marker of infection or whether its concentrations in aSAH patients are associated with the initial hemorrhage, which would mean that a larger hemorrhage might increase the grade of inflammation, worsening the general state of the patient and consequently increasing the risk of infection. Alternatively, further pathophysiological insights may be obtained by measuring the concentrations of neopterin in patient CSF. As already mentioned by Mathiesen et al., ${ }^{23}$ this polar molecule does not cross the blood-brain barrier, so the direct evaluation of the inflammation produced by the hemorrhage may increase understanding of the relation between inflammation and infection development. ${ }^{23}$

Furthermore, in this cohort, the development of DCI and the amounts of blood measured by the Fisher scale were not correlated with outcome, findings which do not reflect the data in the literature. ${ }^{7,9,18}$

In summary, once these small confounders are elucidated, neopterin could become a useful biomarker to improve the clinical management and outcome in aSAH patients.

\section{Conclusions}

Neopterin is a potential outcome and infection predictor after aSAH. The objective information provided by the measurement of neopterin concentrations immediately after hospital admission and onward may enhance the performance of other clinical methods for patient assessment. To evaluate the clinical utility of this biomarker, the results of the present study should be validated in larger and multicenter studies.

\section{Acknowledgments}

We thank Catherine Fouda and Nadia Walter as well as the nurses of Pitié-Salpêtrière Hospital for their remarkable technical work and in the collection of samples. The collection of samples was funded by the Direction for Clinical Research of the Assistance Publique-Hopitaux de Paris. This work was also supported by "Fondation Pierre Mercier pour la science."

\section{References}

1. Al-Khindi T, Macdonald RL, Schweizer TA: Cognitive and functional outcome after aneurysmal subarachnoid hemorrhage. Stroke 41:e519-e536, 2010

2. Beaudeux JL, Léger P, Dequen L, Gandjbakhch I, Coriat P, Foglietti MJ: Influence of hemolysis on the measurement of $\mathrm{S}-100 \beta$ protein and neuron-specific enolase plasma concentrations during coronary artery bypass grafting. Clin Chem 46:989-990, 2000

3. Bederson JB, Connolly ES Jr, Batjer HH, Dacey RG, Dion JE, Diringer MN, et al: Guidelines for the management of aneurysmal subarachnoid hemorrhage: a statement for healthcare professionals from a special writing group of the Stroke Council, American Heart Association. Stroke 40:994-1025, 2009

4. Calandra T, Cohen J: The international sepsis forum consensus conference on definitions of infection in the intensive care unit. Crit Care Med 33:1538-1548, 2005

5. Carr KR, Zuckerman SL, Mocco J: Inflammation, cerebral vasospasm, and evolving theories of delayed cerebral ischemia. Neurol Res Int 2013:506584, 2013

6. Chadha S, Bhalla P, Gautam H, Chakravarti A, Saini S, Anuradha S, et al: Utility of serum neopterin and serum IL-2 receptor levels to predict absolute CD4 T lymphocyte count in HIV infected cases. Interdiscip Perspect Infect Dis 2013:143648, 2013

7. Chowdhury T, Dash HH, Cappellani RB, Daya J: Early brain injury and subarachnoid hemorrhage: Where are we at present? Saudi J Anaesth 7:187-190, 2013

8. Dhar R, Diringer MN: The burden of the systemic inflammatory response predicts vasospasm and outcome after subarachnoid hemorrhage. Neurocrit Care 8:404-412, 2008

9. Etminan N, Vergouwen MD, Ilodigwe D, Macdonald RL: Effect of pharmaceutical treatment on vasospasm, delayed cerebral ischemia, and clinical outcome in patients with aneurysmal subarachnoid hemorrhage: a systematic review and meta-analysis. J Cereb Blood Flow Metab 31:1443-1451, 2011

10. Frontera JA, Fernandez A, Schmidt JM, Claassen J, Wartenberg KE, Badjatia N, et al: Impact of nosocomial infectious complications after subarachnoid hemorrhage. Neurosurgery 62:80-87, 2008

11. Fujii M, Yan J, Rolland WB, Soejima Y, Caner B, Zhang JH: Early brain injury, an evolving frontier in subarachnoid hemorrhage research. Transl Stroke Res 4:432-446, 2013

12. Haug T, Sorteberg A, Sorteberg W, Lindegaard KF, Lundar T, Finset A: Cognitive outcome after aneurysmal subarachnoid hemorrhage: time course of recovery and relationship to clinical, radiological, and management parameters. Neurosurgery 60:649-657, 2007

13. Hong CM, Tosun C, Kurland DB, Gerzanich V, Schreibman D, Simard JM: Biomarkers as outcome predictors in subarachnoid hemorrhage - a systematic review. Biomarkers 19:95-108, 2014

14. Iosif C, Di Maria F, Sourour N, Degos V, Bonneville F, Biondi A, et al: Is a high initial World Federation of Neurosurgery (WFNS) grade really associated with a poor clinical outcome in elderly patients with ruptured intracranial aneurysms treated with coiling? J Neurointerv Surg 6:286-290, 2014

15. Kimball MM, Velat GJ, Hoh BL: Critical care guidelines on the endovascular management of cerebral vasospasm. Neurocrit Care 15:336-341, 2011

16. Korinek AM, Baugnon T, Golmard JL, van Effenterre R, Coriat P, Puybasset L: Risk factors for adult nosocomial meningitis after craniotomy: role of antibiotic prophylaxis. Neurosurgery 62 (Suppl 2):532-539, 2008

17. Lagares A, Gómez PA, Alen JF, Lobato RD, Rivas JJ, Alday $\mathrm{R}$, et al: A comparison of different grading scales for predicting outcome after subarachnoid haemorrhage. Acta Neurochir (Wien) 147:5-16, 2005

18. Latorre JG, Lodi Y, El-Zammar Z, Devasenapathy A: Is asymptomatic vasospasm associated with poor outcome in subarachnoid hemorrhage? Neurohospitalist 1:165-171, 2011

19. Lhee HY, Kim H, Joo KJ, Jung SS, Lee KB: The clinical significance of serum and urinary neopterin levels in several renal diseases. J Korean Med Sci 21:678-682, 2006

20. Li ZQ, Wang QH, Chen G, Quan Z: Outcomes of endovascular coiling versus surgical clipping in the treatment of ruptured intracranial aneurysms. J Int Med Res 40:2145-2151, 2012

21. Linn FHH, Rinkel GJE, Algra A, van Gijn J: Incidence of subarachnoid hemorrhage: role of region, year, and rate of computed tomography: a meta-analysis. Stroke 27:625-629, 1996

22. Maddahi A, Povlsen GK, Edvinsson L: Regulation of enhanced cerebrovascular expression of proinflammatory mediators in experimental subarachnoid hemorrhage via the 
mitogen-activated protein kinase kinase/extracellular signalregulated kinase pathway. J Neuroinflammation 9:274, 2012

23. Mathiesen T, Fuchs D, Wachter H, von Holst H: Increased CSF neopterin levels in subarachnoid hemorrhage. J Neurosurg 73:69-71, 1990

24. McMahon CJ, Hopkins S, Vail A, King AT, Smith D, Illingworth KJ, et al: Inflammation as a predictor for delayed cerebral ischemia after aneurysmal subarachnoid haemorrhage. J Neurointerv Surg 5:512-517, 2013

25. Molyneux AJ, Kerr RS, Yu LM, Clarke M, Sneade M, Yarnold JA, et al: International subarachnoid aneurysm trial (ISAT) of neurosurgical clipping versus endovascular coiling in 2143 patients with ruptured intracranial aneurysms: a randomised comparison of effects on survival, dependency, seizures, rebleeding, subgroups, and aneurysm occlusion. Lancet 366:809-817, 2005

26. Murr C, Widner B, Wirleitner B, Fuchs D: Neopterin as a marker for immune system activation. Curr Drug Metab 3:175-187, 2002

27. Oertel M, Schumacher U, McArthur DL, Kästner S, Böker DK: S-100B and NSE: markers of initial impact of subarachnoid haemorrhage and their relation to vasospasm and outcome. J Clin Neurosci 13:834-840, 2006

28. Oshiro EM, Walter KA, Piantadosi S, Witham TF, Tamargo RJ: A new subarachnoid hemorrhage grading system based on the Glasgow Coma Scale: a comparison with the Hunt and Hess and World Federation of Neurological Surgeons Scales in a clinical series. Neurosurgery 41:140-148, 1997

29. Provencio JJ: Inflammation in subarachnoid hemorrhage and delayed deterioration associated with vasospasm: a review. Acta Neurochir Suppl 115:233-238, 2013

30. Robin X, Turck N, Hainard A, Tiberti N, Lisacek F, Sanchez JC, et al: pROC: an open-source package for $\mathrm{R}$ and $\mathrm{S}+$ to analyze and compare ROC curves. BMC Bioinformatics 12:77, 2011

31. Sanchez-Peña P, Pereira AR, Sourour NA, Biondi A, Lejean L, Colonne C, et al: S100B as an additional prognostic marker in subarachnoid aneurysmal hemorrhage. Crit Care Med 36:2267-2273, 2008

32. Schievink WI: Intracranial aneurysms. N Engl J Med 336:28-40, 1997

33. Simon L, Gauvin F, Amre DK, Saint-Louis P, Lacroix J: Serum procalcitonin and C-reactive protein levels as markers of bacterial infection: a systematic review and meta-analysis. Clin Infect Dis 39:206-217, 2004
34. Suarez JI, Tarr RW, Selman WR: Aneurysmal subarachnoid hemorrhage. N Engl J Med 354:387-396, 2006

35. Tiberti N, Lejon V, Hainard A, Courtioux B, Robin X, Turck $\mathrm{N}$, et al: Neopterin is a cerebrospinal fluid marker for treatment outcome evaluation in patients affected by Trypanosoma brucei gambiense sleeping sickness. PLoS Negl Trop Dis 7:e2088, 2013

36. Turck N, Vutskits L, Sanchez-Pena P, Robin X, Hainard A, Gex-Fabry M, et al: A multiparameter panel method for outcome prediction following aneurysmal subarachnoid hemorrhage. Intensive Care Med 36:107-115, 2010

37. Wang HC, Lin WC, Yang TM, Lin YJ, Tsai NW, Cheng KY, et al: The association between symptomatic delayed cerebral infarction and serum adhesion molecules in aneurysmal subarachnoid hemorrhage. Neurosurgery 68:1611-1617, 2011

38. Weiss N, Sanchez-Peña P, Roche S, Beaudeux JL, Colonne C, Coriat P, et al: Prognosis value of plasma S100B protein levels after subarachnoid aneurysmal hemorrhage. Anesthesiology 104:658-666, 2006

39. Yoshimoto Y, Tanaka Y, Hoya K: Acute systemic inflammatory response syndrome in subarachnoid hemorrhage. Stroke 32:1989-1993, 2001

\section{Disclosure}

The authors report no conflict of interest concerning the materials or methods used in this study or the findings specified in this paper.

\section{Author Contributions}

Conception and design: Sanchez, Azurmendi, Tiberti. Acquisition of data: Azurmendi. Analysis and interpretation of data: Azurmendi, Tiberti, Kapandji, Turck. Drafting the article: Sanchez, Azurmendi, Tiberti. Critically revising the article: Sanchez, Degos, Tiberti. Reviewed submitted version of manuscript: Sanchez, Turck. Approved the final version of the manuscript on behalf of all authors: Sanchez. Statistical analysis: Azurmendi, Degos, Tiberti, Turck. Study supervision: Sanchez, SanchezPeña, Sarrafzadeh, Puybasset, Turck.

\section{Correspondence}

Jean-Charles Sanchez, Translational Biomarker Group, Department of Human Protein Sciences, Rue Michel Servet 1, CH-1211 Genève 4, Switzerland. email: jean-charles.sanchez@unige.ch. 